\title{
A masked priming ERP study of letter processing using single letters and false fonts
}

\author{
Priya Mitra and Donna Coch \\ Dartmouth College, Hanover, New Hampshire
}

\begin{abstract}
Previous event-related potential (ERP) research on letter processing has suggested that a P150 reflects lowlevel, featural processing, whereas a P260 reflects high-level, abstract letter processing. In order to investigate the specificity of these effects, ERPs were recorded in a masked priming paradigm using matching and nonmatching pairs of letters (e.g., g-g, g-j) and false fonts (e.g., 8-8, 8-.7). If the P150 priming effect indexes featural processing, there should be no effect of condition on the P150, since the letters and false fonts shared visual features. If the P260 priming effect indexes the processing of abstract letter representations, it should be evident only in the letter condition. As was expected, the P150 priming effect was similar for letters and false fonts; however, the P260 priming effect was also similar between conditions. Thus, the P260 priming effect may not be sensitive to abstract letter processing per se, or such processing may be extremely abstract.
\end{abstract}

Although a number of studies have investigated word processing in the human brain (e.g., Cohen et al., 2000; McCandliss, Cohen, \& Dehaene, 2003; Petersen, Fox, Snyder, \& Raichle, 1990), few studies have explored single letters as the parts that make up those words. However, a handful of behavioral studies have investigated letter processing in terms of case (uppercase vs. lowercase; e.g., Boles \& Eveland, 1983; Bowers, Vigliocco, \& Haan, 1998; Haber \& Cole, 1980) and identity (real letters vs. nonletter stimuli, such as symbols, numbers, or letter-like characters; e.g., Gibson, Gibson, Pick, \& Osser, 1962; Lachmann \& van Leeuwen, 2004). The results of such studies have been used to develop a theoretical model of single-letter processing (Jacobs \& Grainger, 1991) that has been extended recently to include event-related potential (ERP) data outlining the time course of the various proposed stages of letter processing (Petit, Midgley, Holcomb, \& Grainger, 2006). These ERP data suggest that a P150 priming effect reflects featural processing, whereas a P260 priming effect reflects abstract letter processing (Petit et al., 2006). Here, we used a masked priming ERP paradigm with real letters and letter-like false font stimuli to further investigate the nature of these priming effects. We hypothesized that if the $\mathrm{P} 150$ priming effect indexes featural processing, letters and false fonts created from letter features should elicit similar P150 priming effects. In contrast, if the P260 priming effect indexes abstract letter processing, only real letter stimuli should elicit this effect.

\section{Models of Letter Processing and ERP Evidence}

In the Jacobs and Grainger (1991) model of letter processing, an extension of an interactive activation model for word processing (McClelland \& Rumelhart, 1981), activations occur at three levels: a feature level, a casespecific level, and an abstract (name) level. Others have suggested similar models of letter processing including visual featural, case-specific, and abstract levels (e.g., Arguin \& Bub, 1995; Selfridge, 1959); with regard to the latter, some have proposed both font-free but casespecific abstract letter representations and font-free, casefree abstract letter representations (Brunsdon, Coltheart, \& Nickels, 2006). Computational models have also been constructed that encode letters independently of case, in terms of abstract letter identities (Polk \& Farah, 1997). However, these models do not speak to the neural time course of letter processing or the online automaticity of letter processing in fluent readers.

With real-time temporal sensitivity, the recording of ERPs is an excellent method for investigating the time course of letter processing. In a masked priming study, Petit et al. (2006) presented prime-target pairs of letters that were either matched or unmatched for both name and case. The authors reported that the ERP waveform elicited by letter reading included three different components indexing the early processing of letters. The amplitude of the first of these components, the P150 (120-180 msec), was modulated by the visual similarity of the prime and target (uppercase and lowercase versions of a letter that were more similar [e.g., $C c$ ] elicited a smaller P150 priming effect than did pairs that were less similar [e.g., $D d]$ ), indicating sensitivity to the featural level of processing. The sensitivity of the N/P150 to feature-level processing and location has also been reported in studies with word stimuli (e.g., Chauncey, Holcomb, \& Grainger, 2008; Dufau, Grainger, \& Holcomb, 2008; Holcomb \& Grainger, 
2006). The second component, the P200 (180-220 msec), appeared to reflect activation of case-specific letter representations; pairs that were matched for both name and case elicited the smallest P200 priming effect. The third component, the P260 (220-300 msec), was identified as indexing abstract letter representations; pairs that were matched for name, regardless of case, elicited a smaller P260 than did pairs that were not matched for name. Petit and colleagues (2006, p. 680) noted the consistency of these findings with a model of letter processing proposing early featural analysis, the results of which converge on case-specific representations, which feed into more abstract letter representations, which in turn feed into higher level systems that utilize letter identities (see, e.g., Jacobs \& Grainger, 1991).

To our knowledge, there have been few other ERP studies of single-letter processing. One early ERP study with single letters also reported sensitivity of the P1 to vowels in print (Mecacci \& Salmaso, 1984), and a more recent ERP study using single-letter stimuli showed that the N170 elicited by letters was more negative than that elicited by both nonletter stimuli and Chinese characters in monolingual English speakers, whereas the N170 was similar for letters from the Roman alphabet and Chinese characters in Chinese-English bilinguals, suggesting that the N170 may be an index of expertise in letter processing (Wong, Gauthier, Woroch, DeBuse, \& Curran, 2005). In the context of a memory task, a positive potential peaking around $170 \mathrm{msec}$ indexed lower level visual analysis, a potential peaking between 200 and $400 \mathrm{msec}$ indexed a memory-matching process independent of a physical match, and processing within a later window was sensitive to set size (Shin, Fabiani, \& Gratton, 2006). Considering the paucity of ERP studies of letter processing, here we used both single-letter and false font stimuli to further investigate the model proposed in Jacobs and Grainger (1991) and Petit et al. (2006); specifically, we investigated the theoretical first stage of letter processing, visual feature analysis as indexed by the P150 priming effect, and the third stage, processing of abstract letter representations as indexed by the P260 priming effect.

\section{Behavioral Studies of Letter Processing: \\ Features and Abstract Representations}

Featural, case, and abstract levels of analysis also have been investigated in behavioral studies of letter processing. Indeed, many of the extant behavioral studies of letter processing have focused on the surface characteristics or orthographic features of letters, such as case, font, size, orientation, confusability, or similarity (e.g., Ambler \& Proctor, 1976; Appelman \& Mayzner, 1982; Briggs \& Hocevar, 1975; Dick, 1970; Dunn-Rankin, Leton, \& Shelton, 1968; Gervais, Harvey, \& Roberts, 1984; Pelli, Burns, Farell, \& Moore-Page, 2006). There is evidence that fluent readers process multiple features of letters simultaneously. For example, in a study in which one condition required detection of a single feature to decide whether pairs of letters were in the same case and another condition required detection of multiple features to de- cide whether pairs of letters had the same name, response latencies were similar between conditions (Cole \& Haber, 1980; see also Townsend \& Ashby, 1982). Confusability matrices likewise suggest that letters are processed in terms of features, since letters with similar features and spatial arrangements are more often confused (e.g., Briggs \& Hocevar, 1975; Dunn-Rankin et al., 1968; Gervais et al., 1984). In studies of the learning of foreign alphabets, the findings have also been consistent with a theoretical feature level of processing; indeed, the results have suggested that letter identification may be determined by detection "of about 7 visual features" (Pelli et al., 2006, p. 4646).

Beyond the level of visual features, behavioral researchers have used letter case to investigate abstract letter representations. Although $D$ and $d$ are orthographically distinct and vary by visual features, they both map onto the same name (dee), a more abstract level of letter representation. In adults, the accuracy and latency of letter-naming responses are unaffected by changes in case in a masked priming paradigm with pairs of single letters, even when the visual features of the letters are dissimilar (Bowers et al., 1998), suggesting that letter naming is, at least in part, dependent on case-insensitive representations. In simple matching tasks, identical letters $(A A)$ are matched more quickly than letters that are not identical orthographically but have the same name (Aa; e.g., Boles \& Eveland, 1983; Posner \& Boies, 1969; Posner \& Keele, 1967). According to Haber and Cole (1980), "the reaction time differences between visual and name matches suggest that the visual representations are established more rapidly or take less time to process than name matches" (p. 183), consistent with at least two separable levels of letter processing: one (earlier) based on visual features and another (later) based on more abstract name information. Taken together, these findings from studies in which case was manipulated consistently suggest a level of case-insensitive, abstract letter representation distinct from a featural level of letter processing. Bigsby (1988) contended that cross-case naming studies index not a name code but "a non-visual, non-phonological, case-independent, font-independent, abstract representation: the abstract letter identity code" (p. 455).

\section{Comparisons of Letter and Nonletter Stimuli}

Although the results of such cross-case comparisons suggest an abstract level of letter processing, they do not address the specificity of such processing; studies that have compared letters and nonletter control stimuli are better designed to address this issue. For example, studies have used digits, common keyboard (ASCII) symbols, and novel "letter-like" shapes as nonletter controls (e.g., Arguin \& Bub, 1995; Burgund, Schlaggar, \& Petersen, 2006; Gibson et al., 1962; Jacobs \& Grainger, 1991; Maurer, Brem, Bucher, \& Brandeis, 2005; Petersen et al., 1990; Petit \& Grainger, 2002; van Leeuwen \& Lachmann, 2004; Wong et al., 2005; Ziegler, Ferrand, Jacobs, Rey, \& Grainger, 2000). In the context of let- 
ter strings, replacing single letters with similarly shaped digits or keyboard symbols in words does not affect response latency in a lexical decision task, but similar substitutions in pseudowords do (Carreiras, Dunabeitia, \& Perea, 2007; Perea, Dunabeitia, \& Carreiras, 2008). This pattern may speak to the automaticity of fluent word processing and, therefore, the necessity of studying individual letters, rather than letter strings, in order to understand letter processing.

Studies in which uppercase and lowercase letters and real letter and nonletter stimuli have been compared have used both naming tasks (e.g., Arguin \& Bub, 1995; Bowers et al., 1998; Martens \& de Jong, 2006; Ziegler et al., 2000) and letter/nonletter classification tasks (e.g., Arguin \& Bub, 1995; van Leeuwen \& Lachmann, 2004; Ziegler et al., 2000). In one study in which both types of tasks were used with both letters and false fonts, the degree of featural overlap between prime and target modulated the priming effect in a letter-naming task more so than in a letter/nonletter classification task (Petit \& Grainger, 2002). This pattern, consistent with the literature reviewed above, suggests that these two tasks (classification and naming) might be indexing two separable levels of letter processing, one sensitive to case and one insensitive to case.

However, a potential confound in studies in which nonletter control stimuli such as digits or ASCII symbols have been used is that most of these stimuli are familiar to most readers and such stimuli have names and may, like letters, have abstract representations; indeed, Jacobs and Grainger (1991) reported identity priming for ASCII symbols, suggesting that "subjects possess some kind of internal representation of [such] nonletters" (p. 49). In order to control for this confound, researchers have created various false fonts: novel, letter-like characters that share features with real letters (e.g., Burgund et al., 2006; Gibson et al., 1962; James, James, Jobard, Wong, \& Gauthier, 2005; Petersen et al., 1990; Petit \& Grainger, 2002; Vinckier et al., 2007; Wong et al., 2005). In matching tasks, latencies for same responses are longer for false font pairs than for letter pairs (Lachmann \& van Leeuwen, 2004), and latencies are similarly longer for isolated false fonts than for letters in a categorical decision task in adults (van Leeuwen \& Lachmann, 2004). These findings suggest that false font identity is more difficult to process, perhaps because "letters form a natural category, and are represented in a more efficient way" (Lachmann \& van Leeuwen, 2004, p. 248); that is, letters have an abstract letter identity code (Bigsby, 1988), whereas false fonts do not.

\section{The Present Study}

To our knowledge, there is no extant masked priming ERP study comparing the processing of single letters and false fonts; such a study would speak to the specificity of the theoretical letter-processing system proposed by Jacobs and Grainger (1991) and Petit et al. (2006). The present study is an expansion upon Petit et al. and was designed to further evaluate the $\mathrm{P} 150$ priming effect as an index of featural processing and the $\mathrm{P} 260$ priming effect as an index of the processing of abstract letter representations. We used both single lowercase letter stimuli and false font characters in a masked-priming paradigm similar to that used by Petit et al. with a letter classification task, in order to determine the specificity of these effects to letters. ${ }^{1}$ As was reviewed above, Petit et al. used both lowercase and uppercase single-letter stimuli in a masked priming paradigm with a randomly cued letter identification task; on the basis of their findings, they theorized that processing within the P150 time window in this paradigm reflected visual feature analysis, that processing within the P200 epoch was case specific, and that processing within the P260 time window indexed abstract letter identities. Because our design did not include case as a factor, we focused on the P150 and P260 priming effects. Overall, we did not manipulate letter name (false fonts had no names) and letter case (we used only lowercase letters), as in Petit et al., but conceptually mapped our design to the theory developed by Jacobs and Grainger and by Petit et al.

In Petit et al. (2006), the P150 priming effect was larger for visually dissimilar prime-target letter pairs and smaller for visually similar prime-target letter pairs, leading to the theoretical conclusion that the P150 priming effect was sensitive to visual features. Here, we hypothesized that if the $\mathrm{P} 150$ priming effect indexes featural processing, there should be no effect of letter versus false font condition on the P150 effect, since the letters and false fonts shared visual features. That is, we predicted that letter targets that did not match primes (e.g., $b$ in the prime-target pair $a-b$ ) would elicit more positive waves in the P150 time window than would letter targets that did match primes (e.g., $a-a$ ) and that the same pattern would be observed in the false font condition. For the P260 priming effect, Petit et al. reported that the effect of name consistency across prime-target letter pairs was significant regardless of case, leading to the theoretical conclusion that the P260 effect was sensitive to abstract, case-independent letter identities. Here, we hypothesized that if the P260 priming effect indexes the processing of abstract letter representations, it should be evident only in the letter condition, and not in the false font condition, since the participants would never have seen the false font stimuli before and would, therefore, not have formed names for or abstract mental representations of these characters. That is, we reasoned that if processing within the 220- to 300 -msec epoch in a masked priming paradigm with single-letter stimuli indexes abstract letter processing, as reflected in the $\mathrm{P} 260$ priming effect (as was proposed by Petit et al., 2006), letter targets that did not match primes would elicit more positive waves in the P260 time window than would letter targets that did match primes, but no similar P260 priming effect should be evident for false font stimuli, which have no names or abstract identities. To our knowledge, this is the first investigation of the time course of letter perception to systematically compare single-letter and false font processing within this theoretical model. 


\section{METHOD}

\section{Participants}

The participants were 10 female and 10 male adults $18.2-27.1$ years of age $(M=20.5, S D=2.6)$. All were right-handed (Edinburgh Handedness Inventory; Oldfield, 1971), with normal or corrected-to-normal vision (20/30 or better on a standard Snellen chart) and no self-reported history of neurological dysfunction or language or reading disorders. Also by self-report, the participants were not literate in or regularly exposed to any orthography other than the Roman alphabet. All were volunteers who were paid \$20 for their participation.

\section{Stimuli}

The stimuli consisted of all 26 lowercase letters of the Roman alphabet, presented in Times New Roman font (e.g., $y, m, k, x$ ), and 26 corresponding false fonts (e.g., $\mathcal{\Lambda}, \mathbf{r}, \mathbf{\downarrow}, \uparrow)$. The false fonts, borrowed from Grossi and Coch (2005), were created by isolating the component parts of each lowercase letter and rearranging them into a new character that no longer resembled any of the letters in the Roman alphabet but was nonetheless letter-like (see Figure 1). The set of 26 false font stimuli was therefore controlled for some basic visual characteristics, including elements of the letters (e.g., curves and straight lines), spatial information, and luminescence (Grossi $\&$ Coch, 2005).

The stimuli were presented in prime-target pairs in a forwardand backward-masked priming paradigm. The prime-target pairs made up four stimulus conditions: matching letter pairs, matching false font pairs, nonmatching letter pairs, and nonmatching false font pairs. Half of all the trials were matching prime-target pairs such that each letter target and each false font target was preceded by an identity prime 5 out of the 10 times that it was presented as a target during the entire list. The other half of all trials consisted of nonmatching prime-target pairs in both the letter and false font conditions. These pairs were constructed so that each letter target was randomly paired with a nonmatching letter prime and each false font target was randomly paired with a nonmatching false font prime. The nonmatching pairs in the letter and false font conditions were equivalent (e.g., the nonmatching prime-target letter pair g-j was mirrored by the nonmatching false font pair $\mathbf{8}_{-} .1$ ). The nonmatching prime-target pairs were held constant across each of the 5 nonmatching trials on which a given target was presented in both the letter and false font conditions. Thus, each target (26 letters and 26 false fonts) was repeated 5 times with a matching/identity prime and 5 times with a nonmatching/nonidentity prime, for a total of 520 trials.

\section{Procedure}

All the procedures were explained to the participants before the experiment, any questions were addressed, and informed consent was obtained. The participants were then fitted with an elastic electrode cap (Electro-Cap International) for the purpose of recording

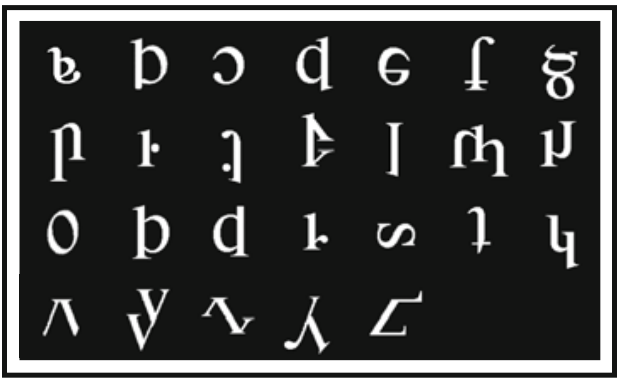

Figure 1. The false font alphabet (originally used in Grossi \& Coch, 2005).
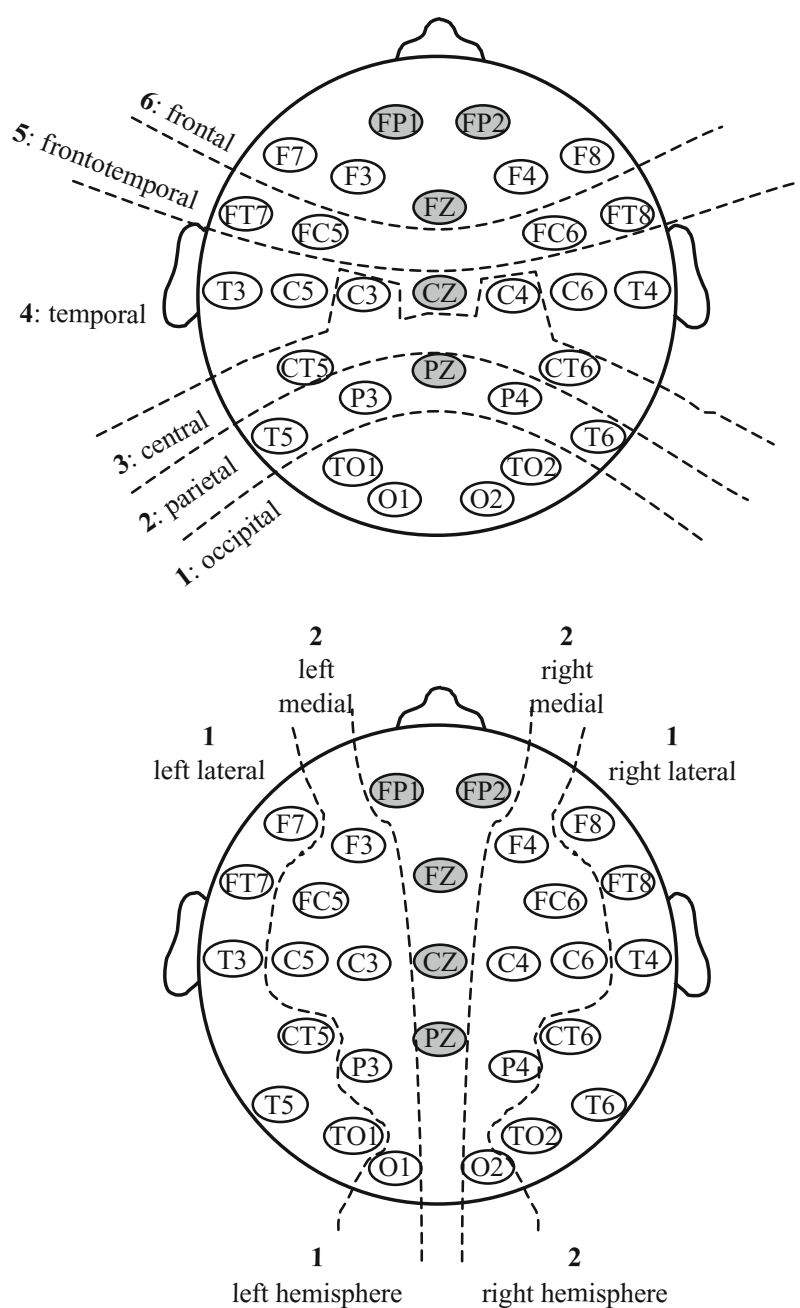

Figure 2. Schematic representation of the electrode montage and the factors used in the analyses. At the top, six levels of the anterior/posterior factor are illustrated. At the bottom, two levels of the lateral/medial factor and two levels of the hemisphere factor are indicated. Electrodes in gray were not used in the primary analyses reported in the text.

electroencephalograms (EEGs). Active electrodes included FP1/2, F7/8, FT7/8, F3/4, FC5/6, C3/4, C5/6, T3/4, CT5/6, P3/4, T5/6, $\mathrm{TO} 1 / 2$, and $\mathrm{O} 1 / 2$; recordings were also taken at the midline sites $\mathrm{Fz}, \mathrm{Pz}$, and $\mathrm{Cz}$, but data from these sites are not analyzed here (see Figure 2). Five loose electrodes were located on the left and right mastoids, at the outer canthi of the left and right eyes, and beneath the lower right eye; mastoid electrodes were used for reference, and eye electrodes were used to identify blinks (in conjunction with recordings from FP1/2) and eye movements. Scalp and mastoid electrode impedances were maintained below $3 \mathrm{k} \Omega$, and eye electrode impedances below $10 \mathrm{k} \Omega$. Once electrode preparation was complete, the participants were moved to a sound-attenuating and electrically shielded booth for ERP testing.

In the booth, the stimuli were presented on a 19-in. monitor approximately $131 \mathrm{~cm}$ in front of the participants, so that both letter and false font stimuli subtended an average of $0.68^{\circ}$ of vertical visual angle and $0.43^{\circ}$ of horizontal visual angle. The primes and targets were presented in the center of the screen, as white characters on a black background. Letter pairs and false font pairs were intermixed in one list, and presentation order of the 520 pairs was 


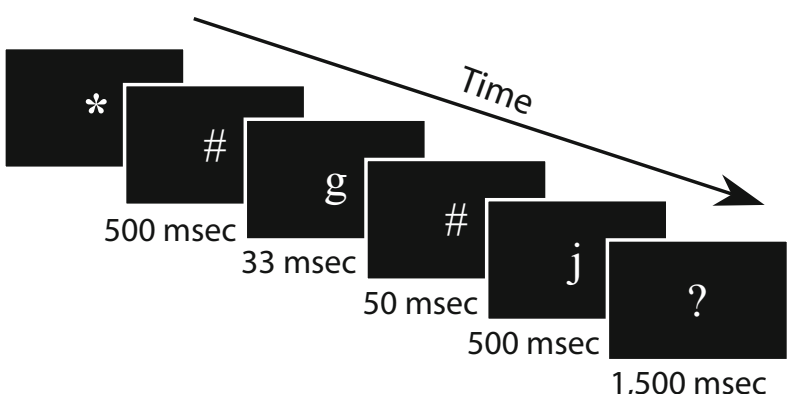

Figure 3. A representative trial in the nonmatching letter condition. A red asterisk/fixation point remained on the screen until a buttonpress from the participant started the trial. The trials consisted of a 500-msec premask (\#), 33-msec prime (g), 50-msec postmask (\#), 500-msec target (j), and 1,500-msec question mark cuing the letter/nonletter buttonpress response. Stimulus presentation times took into account the refresh rate of the monitor.

randomized for each participant. Each trial proceeded as follows (refer to Figure 3). A red asterisk presented in the center of the screen served as a fixation point and marked the beginning of each new trial. The experiment was self-paced, and after the appearance of the red asterisk, the participants pressed a button to begin the trial. Following the buttonpress, a premask (consisting of the pound sign, \#) appeared for $500 \mathrm{msec}$, followed by the prime for $33 \mathrm{msec}$, by a postmask (\#) for $50 \mathrm{msec}$, and finally by the target stimulus for $500 \mathrm{msec}$. After the target disappeared from the screen, a blue question mark appeared, and the participants were asked to respond as to whether the target character was a real letter in its typical orientation or not by pressing the corresponding button on a response device. Response hand was counterbalanced across participants. A practice session preceded the start of data collection, and the experimental portion of the procedure lasted approximately $45 \mathrm{~min}$, including breaks. The total duration of the study was about $2 \mathrm{~h}$ per participant.

EEG was amplified with SA Instrumentation bioamplifiers (bandpass, 0.01-100 Hz) and digitized online (sampling rate, $4 \mathrm{msec}$ ). Online recordings were referenced to the right mastoid and later rereferenced to averaged left/right mastoids in the final data averaging. For the duration of the trial, from the time the participants pressed a button to start a trial at the red asterisk to the time the target disappeared and the blue question mark appeared on the screen, the participants were instructed not to blink or move, in order to reduce artifacts. Standard artifact rejection parameters were initially employed, and the data were subsequently analyzed on an individual basis for artifact rejection. Only trials that were responded to correctly and were free from artifacts were included in the analyses.

\section{Data Analyses}

ERPs were time-locked to presentation of the target stimulus in each pair. Averaged ERPs were quantified by calculating the mean amplitude values for the targets of matching and nonmatching pairs in both the 120- to $180-\mathrm{msec}$ (P150) and the 220- to 300msec (P260) time windows defined by Petit et al. (2006), using a 100 -msec pre-stimulus-onset baseline. For each time window, an omnibus repeated measures ANOVA was performed with withinsubjects factors of condition (two levels: letters, false fonts), match (two levels: matching, nonmatching), anterior/posterior (six levels: frontal [F7/8, F3/4], frontotemporal [FT7/8, FC5/6], temporal [T3/4, $\mathrm{C} 5 / 6]$, centrotemporal [CT5/6, C3/4], temporoparietal [T5/6, P3/4], and temporo-occipital [TO1/2, O1/2]), lateral/medial (two levels), and hemisphere (two levels: left, right) (refer to Figure 2). ${ }^{2}$ A similar ANOVA was performed on peak latency measurements within the 220 - to $300-\mathrm{msec}$ epoch. Peak latency was determined by an auto- mated program that identified the most positive data point within the specified 220- to 300-msec time window, relative to the two preceding and two subsequent data points, to prevent local maxima errors. Significant ERP effects involving condition were followed up with further ANOVAs, and the Greenhouse-Geisser correction was applied to all the within-subjects measures with more than one degree of freedom. All the results were significant at the .05 level unless otherwise noted.

\section{RESULTS}

\section{ERP Task Behavioral Accuracy}

The participants responded to targets with a letter/ nonletter buttonpress for 130 trials each of matching letter pairs, nonmatching letter pairs, matching false font pairs, and nonmatching false font pairs. As was expected, accuracy measures indicated that the participants were highly proficient at the task, with greater than $95 \%$ overall accuracy at identifying real letter targets (see Figure 4). However, the participants were more accurate in the letter $(M=127.15, S D=0.84)$ than in the false font $(M=120.63, S D=1.03)$ condition $[F(1,19)=55.55$, $p<.001$ ], and this varied by match [condition $\times$ match, $F(1,19)=8.05, p<.05]$. Follow-up $t$ tests demonstrated that the participants were slightly more accurate in classifying the targets of nonmatching $(M=127.70$ trials, $S D=3.33)$ than matching $(M=126.60$ trials, $S D=4.31)$ letter pairs $[t(19)=-2.77, p<.05]$, whereas there was no difference in response accuracy between matching and nonmatching targets in the false font condition $[t(19)=$ $1.15, p=.264]$.

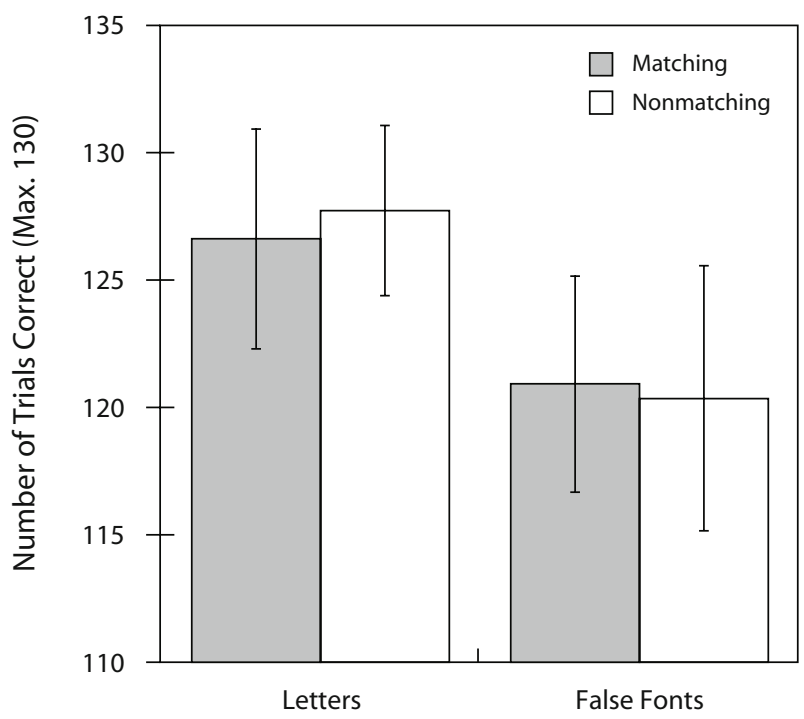

Condition

Figure 4. Behavioral accuracy was high ( $>95 \%$ overall) for both the letter and false font conditions. A significant interaction between condition and match indicated that the participants were more accurate for nonmatching than for matching letter targets, with no effect of match in the false font condition. Bars indicate standard deviations, and the maximum number of correct trials per condition was 130 . 


\section{ERP Mean Amplitude: 120-180 msec (P150)}

Although the main effect of condition was not significant $[F(1,19)=0.32, p=.576]$, the mean amplitude of the P150 appeared to be slightly larger for false fonts than for letters at the most posterior sites [condition $\times$ anterior/ posterior, $F(5,95)=5.50, p<.05]$. The main effect of match also was not significant $[F(1,19)=0.476, p=$ .499], nor was the interaction between condition and match $[F(1,19)=1.10, p=.308]$. However, the effect of match varied across the scalp, so that, particularly at medial sites, nonmatching targets elicited more positive activity than did matching targets at more posterior sites, whereas at more anterior sites, nonmatching targets elicited less positive activity than did matching targets [match $\times$ anterior/ posterior, $F(5,95)=42.40, p<.001$; match $\times$ lateral $/$ medial, $F(1,19)=24.88, p<.001$; match $\times$ anterior/ posterior $\times$ lateral $/$ medial, $F(5,95)=12.98, p<.001$; refer to Figures $5 \mathrm{~A}$ and $5 \mathrm{~B}$ and Figure $6 \mathrm{C}]$.

\section{ERP Mean Amplitude: 220-300 msec (P260)}

A significant effect of condition $[F(1,19)=20.05, p<$ $.001]$ indicated that $\mathrm{P} 260$ mean amplitude was larger in the false font $(M=2.29 \mu \mathrm{V}, S D=1.39)$ than in the letter $(M=1.94 \mu \mathrm{V}, S D=1.44)$ condition. This varied across the scalp such that the greater positivity for false fonts was especially pronounced laterally and at more posterior sites [condition $\times$ lateral $/$ medial, $F(1,19)=5.55, p<.05$; condition $\times$ anterior/posterior $\times$ lateral $/$ medial, $F(5,95)=$ $17.50, p<.001]$. As was expected, there was also a typical priming effect: Targets following matching primes $(M=$ $1.95 \mu \mathrm{V}, S D=1.40$ ) elicited a less positive waveform (smaller P260) than did targets preceded by nonmatching primes $(M=2.28 \mu \mathrm{V}, S D=1.44)$ [match, $F(1,19)=$ $11.88, p<.01]$. A significant interaction between these two factors of condition (letters, false fonts) and match (matching, nonmatching) would provide support for Petit et al.'s (2006) hypothesis regarding the P260 priming effect as an index of abstract letter processing, because it would indicate that the priming effect in the letter condition was different from the priming effect in the false font condition, confirming the specificity of the effect for letter processing. However, the interaction between match and condition was not significant in the present analyses $[F(1,19)=0.63, p=.437$; see Figures 6A and 6B] .

In order to further investigate the condition effects found in the omnibus analysis of mean amplitude measures in the 220- to 300-msec epoch, follow-up ANOVAs were conducted separately for the letter and false font conditions. In the letter condition, the main effect of match was significant $[F(1,19)=12.29, p<.01]$, reflecting the typical priming effect such that targets of nonmatching pairs $(M=2.08 \mu \mathrm{V}, S D=1.52)$ elicited a larger P260 than did those of matching pairs $(M=1.80 \mu \mathrm{V}, S D=1.39)$. This priming effect (i.e., the greater positivity to nonmatching than to matching letter targets) appeared most pronounced at medial and posterior sites [match $\times$ anterior/posterior, $F(5,95)=4.94, p<.05$; match $\times$ anterior/posterior $\times$ lateral/medial, $F(5,95)=2.76, p<.05]$. In the false font condition, the main effect of match also was significant
$[F(1,19)=7.70, p<.05]$, again reflecting a more positive waveform to the nonmatching $(M=2.47 \mu \mathrm{V}, S D=1.40)$ than to the matching $(M=2.10 \mu \mathrm{V}, S D=1.44)$ targets. This difference appeared largest at posterior sites and at medial sites anterior to the most posterior sites [match $\times$ lateral $/$ medial, $F(1,19)=5.26, p<.05$; match $\times$ anterior/ posterior, $F(5,95)=3.28, p=.06$; match $\times$ anterior/ posterior $\times$ lateral $/$ medial, $F(5,95)=4.07, p<.05]$. As a further comparison of the $\mathrm{P} 260$ priming effect between conditions, difference waves were created by subtracting the value of the matching waveform from the value of the nonmatching waveform at each time point, for each participant, at each electrode site, in each condition. Confirming the pattern of findings reported above, mean amplitude of the difference waves measured in the 220to $300-\mathrm{msec}$ epoch was similar for letters and false fonts [condition, $F(1,19)=0.62, p=.442$ ] and did not vary across the scalp by condition (all $p$ values $\geq .176$ ). The voltage maps in Figure 6B illustrate the similarity of the P260 priming effect between conditions.

\section{ERP Peak Latency: 220-300 msec (P260)}

In order to further compare the $\mathrm{P} 260$ priming effects in the letter and false font conditions, an additional omnibus ANOVA was used to investigate the possibility of mean peak latency differences between conditions. The P260 peaked slightly earlier in the letter condition (260.29 msec, $S D=12.55$ ) than in the false font condition (264.78 msec, $S D=9.83)[F(1,19)=5.67, p<.05]$, but this effect of condition on peak latency did not vary by the matching or nonmatching status of the target [condition $\times$ match, $F(1,19)=3.08, p=.095]$. Peak latency analyses of the difference waves confirmed no main effect difference in peak latency for the priming effects in the letter and false font conditions [condition, $F(1,19)=$ $0.41, p=.532]$. However, the interaction among condition, anterior/posterior, and lateral/medial suggested that the P260 priming effect elicited by false fonts tended to peak earlier at anterior sites, whereas the P260 priming effect elicited by letters tended to peak earlier at posterior sites, although this difference was almost exclusively at lateral sites $[F(5,95)=2.69, p<.05]$.

\section{DISCUSSION}

We investigated the featural and abstract levels of letter processing purportedly indexed by the P150 and P260 priming effects, respectively (Petit et al., 2006), in a masked priming paradigm with single-letter and false font stimuli. We hypothesized that if the P150 priming effect reflects featural processing, a similar P150 priming effect would be observed in the letter and false font conditions, since these stimuli shared features. Furthermore, we hypothesized that if the P260 priming effect reflects "processing at the level of abstract letter representations that are independent of letter case" (Petit et al., 2006, p. 680), the P260 priming effect would be observed only in the letter condition and not in the false font condition, since letters should have abstract representations but 
A
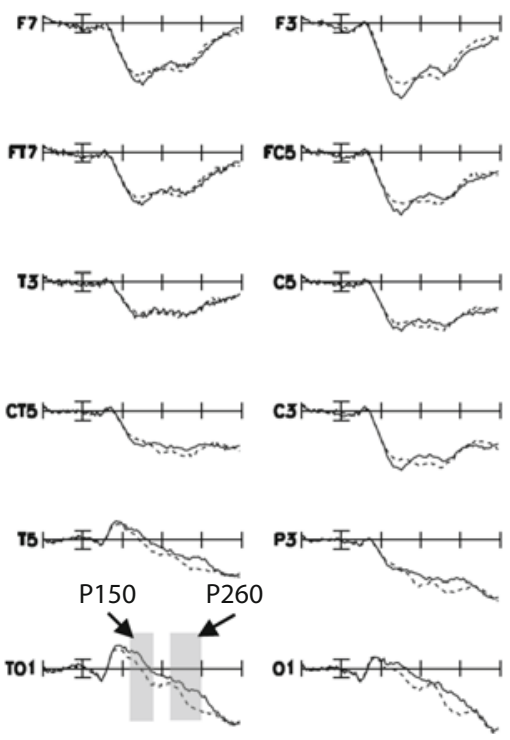

Matching letter targets
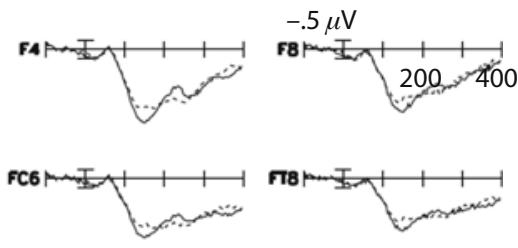

C6rmit 1

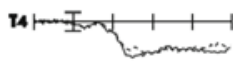

armat 1

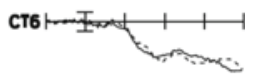

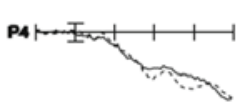

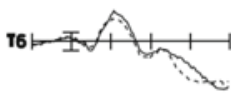

$021-x y+1$

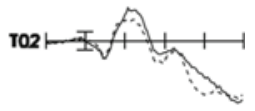

Nonmatching letter targets

\section{B}
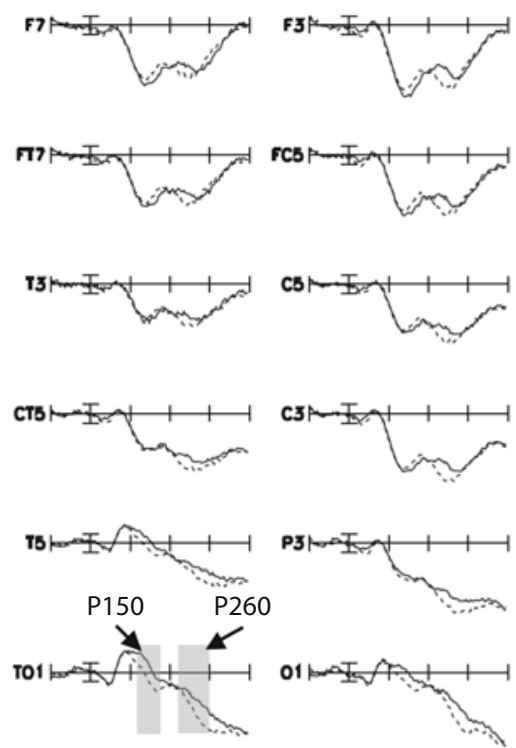

Matching false font targets
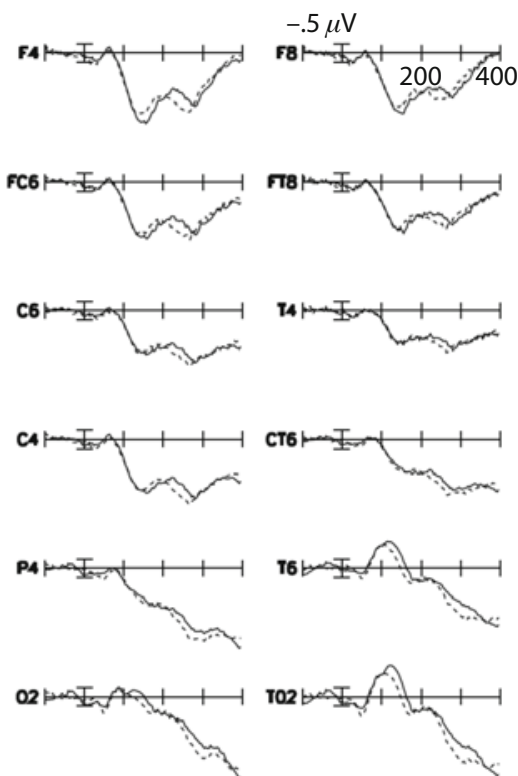

Nonmatching false font targets

Figure 5. Grand average event-related potential waveforms elicited by matching (solid line) and nonmatching (dashed line) letter targets (A) and false fonts targets (B). Within each panel, more anterior sites are toward the top of the figure, and more posterior sites are toward the bottom; left-hemisphere sites are on the left, and righthemisphere sites are on the right; lateral sites are toward the outer edges, and medial sites are toward the middle of the figure; each vertical tick marks $100 \mathrm{msec}$; and negative is plotted up (throughout). The calibration bar marks $0.5 \mu \mathrm{V}$. The approximate time windows for the $\mathrm{P150}$ and $\mathrm{P} 260$ are indicated at site TO1. A priming effect such that nonmatching targets elicited greater positivities than did matching targets is apparent for both the P150 and the P260 for both letters and false fonts. 
A

Letters

Letters

False Fonts

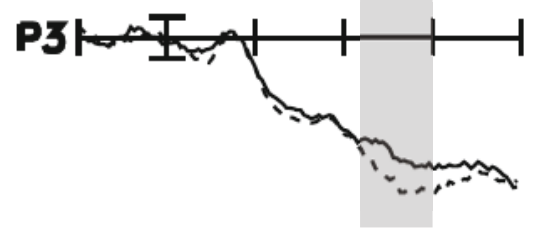

B

Letters
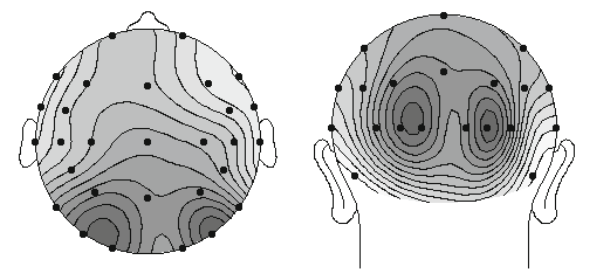

False Fonts
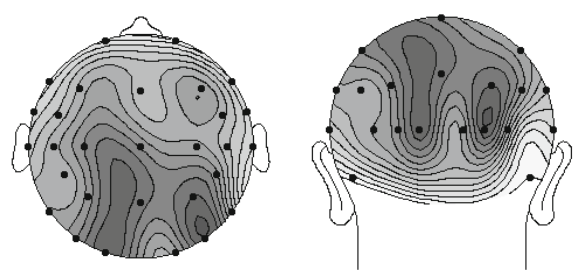

C

Letters
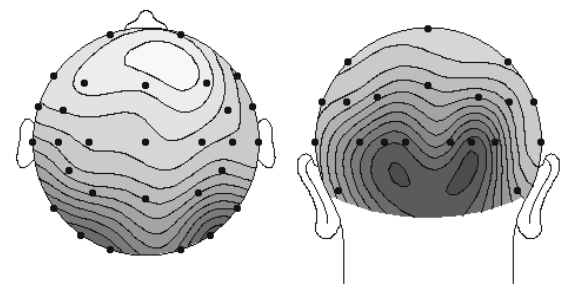

False Fonts

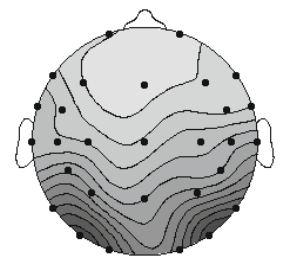

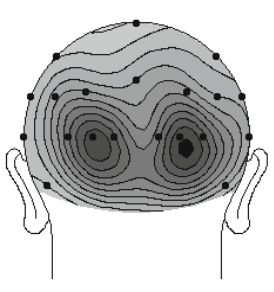

Potential

0.75

0.70

0.64

0.59

0.54

0.48

0.43
0.38

0.32

0.27

0.21

0.16
0.11
0.05

0.05
0.00

$\mu \mathrm{V}$

Figure 6. (A) Zooming in on site P3 (see Figure 2 for full montage) illustrates that the P260 priming effect (larger P260 for nonmatching [dashed lines] than for matching [solid lines] targets; time window shaded gray) was similar for letters and false fonts; indeed, the interaction between condition (letters, false fonts) and match (matching, nonmatching) in the 220- to 300-msec time window was not significant. (B) Topographical voltage maps also illustrate the similarity of the P260 priming effect for letters and false fonts. A spherical spline interpolation (Perrin, Pernier, Bertrand, \& Echallier, 1989) was used to interpolate the potential on the surface of an idealized, spherical head based on the mean amplitude voltages measured from difference waves (reflecting event-related potentials [ERPs] to matching targets subtracted from ERPs to nonmatching targets) at each electrode location within the 220- to $300-\mathrm{msec}$ time window. (C) Topographical voltage maps illustrate the similarity of the $\mathbf{P 1 5 0}$ priming effect for letters and false fonts. 
novel false fonts should not. We observed both a P150 and a P260 priming effect for letters but found that false fonts also elicited both P150 and P260 priming effects that were remarkably similar to the letter effects. This pattern of results is consistent with the hypothesis that the P150 priming effect indexes a visual featural level of processing but appears to be inconsistent with the hypothesis that the P260 priming effect indexes the processing of abstract letter representations.

The $\mathrm{P} 150$ priming effect elicited by letter and false font targets was not significantly different, consistent with the literature indicating that the $\mathrm{P} 150$ is sensitive to a visual featural level of analysis in letter (Petit et al., 2006), word (e.g., Chauncey et al., 2008; Dufau et al., 2008; Holcomb \& Grainger, 2006), and object (e.g., Allison, Puce, Spencer, \& McCarthy, 1999; Eddy, Schmid, \& Holcomb, 2006, for a P190) processing. At the word level, the P150 has been related to processes of visual classification, particularly in terms of familiar visual features (Proverbio, Vecchi, \& Zani, 2004; Schendan, Ganis, \& Kutas, 1998). At the object level, the P150 has also been related to the processing of visual features, since the $\mathrm{P} 150$ to objects and scrambled objects is similar (Allison et al., 1999). In the present study, at the letter level, because the false fonts were composed of the same visual elements as the letters, we predicted that the P150 priming effect would be similar between conditions; this prediction was confirmed. The P150 priming effect observed here was also similar in terms of timing and distribution to the P150 priming effect reported by Petit et al. in their dissimilar cross-case letter comparison: In both studies, nonmatching targets elicited more positive activity than did matching targets at more posterior sites within the $\mathrm{P} 150$ epoch, with a reversal of that effect at anterior sites. The present interpretation of the P150 elicited by single letters and false fonts as reflecting visual featural analysis seems consistent with previous ERP findings, although behavioral studies have suggested that letter and nonletter processing may involve different feature integration strategies (van Leeuwen \& Lachmann, 2004).

Although the P150 priming effect was similar in the letter and false font conditions, false fonts theoretically should not have elicited a P260 priming effect, since these novel stimuli should not have abstract representations. However, the mean amplitude of the P260 priming effect in the 220- to 300 -msec time window was not significantly different for false fonts and real letters, as demonstrated by the lack of a significant interaction between condition (letter or false font) and match (matching or nonmatching prime and target). Moreover, the $\mathrm{P} 260$ priming effect was distributed similarly between conditions: maximal at bilateral, medial, posterior sites, which was clearly illustrated in voltage maps of the difference waves (refer to Figure 6B). Although the results of a number of behavioral and neuroimaging studies in which cross-case (e.g., Boles \& Eveland, 1983; Bowers et al., 1998; Haber \& Cole, 1980; Petit et al., 2006; Shin et al., 2006) and letter/ nonletter (e.g., Flowers et al., 2004; Gibson et al., 1962; James et al., 2005; Lachmann \& van Leeuwen, 2004; Mar- tens \& de Jong, 2006; Petit \& Grainger, 2002; Wong et al., 2005) comparisons have been used to provide evidence for letter-specific abstract representations (e.g., Bigsby, 1988; Brunsdon et al., 2006; James et al., 2005; Petit et al., 2006; Polk \& Farah, 1997; Polk et al., 2002), the present findings with false fonts suggest that the P260 priming effect in a masked priming paradigm may not index such abstract letter processing (cf. Petit et al., 2006).

Although the mean amplitude of the P260 priming effect was not sensitive to differences between the letter and false font conditions, there were differences in the ERP waveforms elicited by letters and false fonts within the 220- to 300-msec epoch, in terms of both amplitude and latency. The overall mean amplitude in this epoch was greater (more positive) for novel false fonts $(M=$ $2.29 \mu \mathrm{V})$ than for familiar letters $(M=1.94 \mu \mathrm{V})$, regardless of the matching or nonmatching status of the prime and target, especially at lateral and posterior sites. This suggests that the amplitude of the P260 component itself may be sensitive to real letter status, although the amplitude of the P260 priming effect is not; it is unclear whether this pattern of results reflects qualitative or quantitative differences in processing, but it seems likely that the differences are a matter of degree and may be indicative of the sensitivity of the P260 to the relative familiarity of the stimuli. In addition to this amplitude difference, the P260 to letters peaked slightly earlier $(M=260.29 \mathrm{msec})$ than the P260 to false fonts $(M=264.78 \mathrm{msec})$, showing that the timing of processes indexed by the P260 is faster to well-known letters than to novel false fonts, consistent with behavioral studies reporting longer latency responses for false fonts than for letters (e.g., Lachmann \& van Leeuwen, 2004; van Leeuwen \& Lachmann, 2004). However, the latency difference was very small overall: just $4.49 \mathrm{msec}$, slightly greater than the 4-msec sampling rate used in data acquisition. In difference wave analyses, the priming effect for letters was earlier at posterior sites, whereas the priming effect for false fonts was earlier at anterior sites, particularly at lateral sites. This pattern of latency findings is consistent with the suggestion that the P260 is sensitive to letter status and provides some support for the hypothesis that the P260 priming effect is sensitive to legal letter status and, in some way, specific to abstract letter representations - but in terms of timing and distribution of peak latency, rather than amplitude.

There were also differences between letters and false fonts in terms of behavioral accuracy in the ERP letter/ nonletter classification task. Not surprisingly, the fluently reading adult participants were highly effective (over 95\% accurate) at differentiating between the familiar real letters and the novel false fonts. However, the participants were more accurate in the letter condition $(M=127.15)$ than in the false font condition $(M=120.63)$, possibly due to the constraints of stimulus construction, rather than to any inherent differences in the difficulty of the task between conditions or in the underlying processing mechanisms. The false fonts were constructed by maintaining each element of a letter as a whole; since some letters in the Roman alphabet are made of single visual elements, 
this limited the transformations that each letter could undergo. Anecdotal evidence from the present study indicated that the false fonts $I$ and $\mathcal{O}$ were especially difficult to distinguish from the letters on which they were based ( $l$ and $o$, respectively). Evidence from a previous behavioral study showed that disorientation of letters does not affect their overall identification or classification (Koriat \& Norman, 1989). Speculatively, if most of the participants perceived these particular false fonts as real letters, this could account for the difference in behavioral performance between conditions; unfortunately, the stimuli were not coded in a way that would allow for an item analysis to investigate this possibility. ${ }^{3}$

Beyond the inclusion of false font stimuli and the use of only lowercase letter stimuli, one of the key differences between the original ERP masked priming study by Petit et al. (2006) and the present study was in terms of task: Petit and colleagues used an infrequent letter identification task (and those trials requiring a response were excluded from analyses), whereas we used a letter/nonletter classification task requiring a response for each trial. Hypothetically, this change of experimental task may have changed the nature of the effect being elicited; such task differences have been shown to affect response latency in behavioral studies (Arguin \& Bub, 1995; Jacobs \& Grainger, 1991; Ziegler et al., 2000). However, although behavioral measures may reflect such task differences, we did not expect that task would affect the early online processing measured in our ERP masked priming paradigm, since such processing should be automatic (e.g., Dehaene et al., 2001; Misra \& Holcomb, 2003). If the priming effects do index automatic processes, any deliberate decisions made, such as the letter/nonletter classification, should not be reflected during the P150 and P260 epochs. Therefore, we contend that the ERP findings in these two studies should be unaffected by the task difference and the results should be comparable. This contention is supported, for example, by the similarity in appearance, timing, and wide distribution of the P260 priming effect elicited by same-case letter stimuli in Petit et al. and our P260 priming effect for lowercase letters. This replication despite task differences suggests that the same underlying, automatic processes are being indexed by both the present letter classification task and the letter identification task used in the original investigation.

Despite the similarities in letter-processing results between the present study and Petit et al. (2006), our findings in the false font condition bring into question the nature of the automatic processing indexed by the $\mathrm{P} 260$ priming effect. Overall, the high degree of similarity between the P260 priming effects elicited in the letter and false font conditions fails to lend adequate support to the contention that completely different underlying processes drive the P260 effects elicited by letters and false fonts. But if the $\mathrm{P} 260$ priming effect reflects processing of abstract letter representations (Petit et al., 2006), why and how could false fonts elicit such processing? If letters form a "natural category" that is represented efficiently by an abstract code (Lachmann \& van Leeuwen, 2004, p. 248), how could false fonts access that code? It is conceivable that the specialized expert processing of letters (assumed in the college student participants, who had no history of reading or language disorder and normal or corrected-tonormal vision) is designed in such a way as to allow for great variability and even inaccuracy in the way that the elements of the letters are assembled and presented. Indeed, that abstract letter processing is not font specific or case specific (e.g., Bigsby, 1988; Petit et al., 2006; Polk et al., 2002) suggests some degree of variability and flexibility within this system; Petit et al. speculated that the P260 indexed "high-level letter representations, which are relatively insensitive to variations in surface form" (p. 680). Because our false font stimuli were well controlled and purposefully letter-like, they may have elicited processing within such a very abstract letter-processing system. ${ }^{4}$ There may also be different levels of abstractness within the abstract letter-processing system (e.g., Brunsdon et al., 2006), only some of which might be more or less responsive to false fonts. The behavioral accuracy data indicated that the participants were able to distinguish real letters from false fonts, but the ERP priming data suggest that this distinction may have occurred sometime after P260 processing.

Another possibility is that the P260 priming effect, rather than indexing abstract letter representations, may simply be sensitive to the basic visual components making up letters, which were identical in the two conditions in the present study. That is, the pattern of results is consistent with an interpretation of the P260 priming effect as an index of visual processing accessed by the visual components that were common to both sets of stimuli, rather than an abstract, linguistic level of processing that was present only in the letter condition. This interpretation is somewhat problematic, because basic visual processing is typically indexed within earlier time windows (e.g., Rugg \& Coles, 1995 ) and the P150 priming effect results are consistent with a similar interpretation; none of our findings speak to the differentiation of processing indexed by the P150 and P260 priming effects. Nonetheless, it remains that the rearranged visual components of letters (false fonts) can elicit the $\mathrm{P} 260$ priming effect, which suggests that the effect is somehow sensitive to the visual elements of the character and perhaps reflects some sort of "higher level" visual processing that occurs subsequent to more basic visual processing. A P250 elicited in face-processing studies has been shown to be sensitive to the specific features of faces regardless of orientation (Marzi \& Viggiano, 2007) and, perhaps, to the configuration of these pieces, but seemingly not to the face as a whole (Milivojevic, Clapp, Johnson, \& Corballis, 2003); speculatively, if this is generalizable to other objects that college students are experts at processing, it is possible that the false fonts were processed as disoriented (or misoriented) letters and that processing peaking around $260 \mathrm{msec}$ is still focused on the component parts, rather than on the composition of those parts into a whole and the legality or identity of the letter. Thus, the abstract processing indexed by the $\mathrm{P} 260$ priming effect may not be specific to letters per se. 
However, there is another interpretation of the present findings that could be more consistent with the nature of the P260 priming effects as proposed by Petit et al. (2006). ${ }^{5}$ It is possible that the P260 priming effects for letters and false fonts were similar because the participants were accessing real letter abstract representations when they processed the false fonts; after repeated exposures, the participants could have been transforming or translating or associating the false font stimuli with their real letter counterparts, and this may have driven the P260 priming effect for false fonts. The slightly longer P260 latency for false font stimuli is consistent with this interpretation, in that it may reflect this transformation process before access to abstract letter representations. In this view, the P260 priming effect may be specific to letters. This hypothesis could be tested by the use of less letter-like false font stimuli (false font stimuli that could not be transformed into real letters) in a similar paradigm, relaxing the orthographic constraints imposed here.

Although the timing of the $\mathrm{P} 150$ and $\mathrm{P} 260$ priming effects suggests that these effects index relatively early visual processing, the neural substrates of such processing are unclear. The topographical distribution of the P150, a large positivity at the most posterior sites and a negativity over anterior sites, was consistent with the distribution of the P150 priming effect reported by Petit et al. (2006). Despite the poor spatial resolution of ERPs, they speculated that the neural generator of the P150 effect was "in or near visual cortical areas responsible for early visual feature processing" (p. 680). Although there are few MEG or fMRI studies of single-letter processing, two MEG responses to letter and symbol strings occur roughly within the P150 time window: one, dominant over left occipitotemporal areas, that is strongest to letter strings and one, dominant over the right extrastriate cortex, that is modulated by string length but not string type (e.g., Tarkiainen, Helenius, Hansen, Cornelissen, \& Salmelin, 1999). The P150 priming effect recorded here appears to be more consistent with the latter, representing "some attribute of object processing that is common to both letter and symbol strings" (Tarkiainen et al., 1999, p. 2128). The P260 priming effect elicited in our masked priming paradigm does not appear to have a correlate in the MEG data.

However, it is difficult to compare studies of letter or symbol strings and studies of single letters, since different occipitotemporal regions have been shown to have distinct patterns of activation for words, letter strings, digit strings, and single letters (e.g., Flowers et al., 2004; James et al., 2005; Joseph, Gathers, \& Piper, 2003; Nobre, Allison, \& McCarthy, 1994; Polk \& Farah, 1998; Polk et al., 2002; Vinckier et al., 2007). For example, the region within the fusiform gyrus that specializes in processing single letters may be slightly lateral to (Flowers et al., 2004) or anterior of (James et al., 2005) the visual word form area. Left-fusiform activation is also greatest in English monolinguals reading single Roman letters, as compared with digits or Chinese characters (James et al., 2005), and fusiform activation is reportedly greater for single letters than for nonletter symbols (Flowers et al., 2004). Furthermore, activation in the left-lateral portion of the middle occipital gyrus appears to be unique to single-letter processing, as compared with symbols and colors (Flowers et al., 2004). Speculatively, these data concerning the neural substrates of letter processing suggest that the P260 priming effect, which was not sensitive to real letter status in our masked priming paradigm, may reflect processing within the visual stream downstream of the fusiform gyrus, consistent with the early timing and posterior topographical distribution of the effect. This speculation is tempered by both the finding that the P260 itself (although not the priming effect) did appear to be sensitive to real letter status and the lack of neuroimaging literature on single-letter processing in priming paradigms, which precludes direct comparison of the fMRI and ERP findings.

In conclusion, in a masked priming ERP paradigm with single letters and false fonts, both the P150 and P260 priming effects appeared to be sensitive to the visual featural aspects of the stimuli; if the $\mathrm{P} 260$ priming effect is an index of abstract letter processing (Petit et al., 2006), the nature of those abstract representations is such that it appears to be inclusive of our false font stimuli. Using single letters in research has the advantage of segmenting print into its simplest parts (e.g., Pelli, Farell, \& Moore, 2003), allowing for study of the basic orthographic elements that compose print. Our findings highlight the importance of critically examining both the nature of abstract letter representations and indices of the processing of such representations by using well-controlled comparison stimuli. Further work is needed to determine the exact nature of the P260 priming effect, other possible neural indices of abstract letter processing, the nature of abstract letter representations and their specificity to letters and letter-like stimuli, and the relations among these. Such research has the potential to illuminate unanswered questions about how we process written language in its simplest, earliest, and most basic orthographic form: single letters.

\section{AUTHOR NOTE}

Portions of this research were presented as a poster at the 2008 Annual Meeting of the Cognitive Neuroscience Society. This research was supported by a Dartmouth College Dean of Faculty Undergraduate Research Grant (Kaminsky Family Fund) and a grant from the Dartmouth College Office of Residential Life and the Class of 1989 (Senior Scholars Program) to the first author. The second author was supported in part by Grant R03HD053362 from the National Institute of Child Health and Human Development. Correspondence concerning this article should be addressed to D. Coch, Department of Education, Dartmouth College, 3 Maynard Street, Raven House, HB 6103, Hanover, NH 03755 (e-mail: donna.coch@dartmouth.edu).

\section{REFERENCES}

Allison, T., Puce, A., Spencer, D. D., \& McCarthy, G. (1999). Electrophysiological studies of human face perception: I. Potentials generated in occipitotemporal cortex by face and non-face stimuli. Cerebral Cortex, 9, 415-430.

Ambler, B. A., \& Proctor, J. D. (1976). The familiarity effect for single-letter pairs. Journal of Experimental Psychology: Human Perception \& Performance, 2, 222-234.

Appelman, I. B., \& Mayzner, M. S. (1982). Application of geometric models to letter recognition: Distance and density. Journal of Experimental Psychology: General, 111, 60-100. 
Arguin, M., \& Bub, D. (1995). Priming and response selection processes in letter classification and identification tasks. Journal of Experimental Psychology: Human Perception \& Performance, 21, 1199-1219.

Bigsby, P. (1988). The visual processor module and normal adult readers. British Journal of Psychology, 79, 455-469.

Boles, D. B., \& Eveland, D. C. (1983). Visual and phonetic codes and the process of generation in letter matching. Journal of Experimental Psychology: Human Perception \& Performance, 9, 657-674.

Bowers, J. S., Vigliocco, G., \& HaAn, R. (1998). Orthographic, phonological, and articulatory contributions to masked letter and word priming. Journal of Experimental Psychology: Human Perception \& Performance, 24, 1705-1719.

Briggs, R., \& Hocevar, D. J. (1975). A new distinctive feature theory for upper case letters. Journal of General Psychology, 93, 87-93.

Brunsdon, R., Coltheart, M., \& Nickels, L. (2006). Severe developmental letter-processing impairment: A treatment case study. Cognitive Neuropsychology, 23, 795-821.

Burgund, E. D., Schlaggar, B. L., \& Petersen, S. E. (2006). Development of letter-specific processing: The effect of reading ability. Acta Psychologica, 122, 99-108.

Carreiras, M., Dunabeitia, J. A., \& Perea, M. (2007). Reading words, numb3r5 and \$ymßol\$. Trends in Cognitive Sciences, 11, 454-455.

Chauncey, K., Holcomb, P. J., \& Grainger, J. (2008). Effects of stimulus font and size on masked repetition priming: An event-related potentials (ERP) investigation. Language \& Cognitive Processes, 23, 183-200.

Cohen, L., Dehaene, S., Naccache, L., Lehéricy, S., DehaeneLambertz, G., Hénaff, M.-A., \& Michel, F. (2000). The visual word form area: Spatial and temporal characterization of an initial stage of reading in normal subjects and posterior split-brain patients. Brain, 123, 291-307.

Cole, R. A., \& HABER, R. N. (1980). Reaction time to letter name or letter case. Acta Psychologica, 44, 281-285.

Dehaene, S., Naccache, L., Cohen, L., Le Bihan, D., Mangin, J.-F., Poline, J.-B., \& Rivière, D. (2001). Cerebral mechanisms of word masking and unconscious repetition priming. Nature Neuroscience, 4, 752-758.

Dick, A. O. (1970). Stimulus variables in single-letter recognition. Psychonomic Science, 20, 195-196.

Dufau, S., Grainger, J., \& Holcomb, P. J. (2008). An ERP investigation of location invariance in masked repetition priming. Cognitive, Affective, \& Behavioral Neuroscience, 8, 222-228.

Dunn-Rankin, P., Leton, D. A., \& Shelton, V. F. (1968). Congruency factors related to visual confusion of English letters. Perceptual \& Motor Skills, 26, 659-666.

Eddy, M., Schmid, A., \& Holcomb, P. J. (2006). Masked repetition priming and event-related brain potentials: A new approach for tracking the time-course of object perception. Psychophysiology, 43, 564568.

Flowers, D. L., Jones, K., Noble, K., VanMeter, J., Zeffiro, T. A., Wood, F. B., \& EDEN, G. F. (2004). Attention to single letters activates left extrastriate cortex. NeuroImage, 21, 829-839.

Gervais, M. J., Harvey, L. O., JR., \& Roberts, J. O. (1984). Identification confusions among letters of the alphabet. Journal of Experimental Psychology: Human Perception \& Performance, 10, 655-666.

Gibson, E. J., Gibson, J. J., Pick, A. D., \& Osser, H. (1962). A developmental study of the discrimination of letter-like forms. Journal of Comparative Physiological Psychology, 55, 897-906.

Grossi, G., \& Coch, D. (2005). Automatic word form processing in masked priming: An ERP study. Psychophysiology, 42, 343-355.

Haber, R. N., \& Cole, R. A. (1980). Evidence for direct visual access to letter identities. Acta Psychologica, 46, 181-192.

Holcomb, P. J., \& Grainger, J. (2006). On the time course of visual word recognition: An event-related potential investigation using masked repetition priming. Journal of Cognitive Neuroscience, 18, 1631-1643.

JaCobs, A. M., \& Grainger, J. (1991). Automatic letter priming in an alphabetic decision task. Perception \& Psychophysics, 49, 43-52.

James, K. H., James, T. W., Jobard, G., Wong, A. C.-N., \& GaUTHIER, I. (2005). Letter processing in the visual system: Different ac- tivation patterns for single letters and strings. Cognitive, Affective, \& Behavioral Neuroscience, 5, 452-466.

Joseph, J. E., Gathers, A. D., \& Piper, G. A. (2003). Shared and dissociated cortical regions for object and letter processing. Cognitive Brain Research, 17, 56-67.

Koriat, A., \& Norman, J. (1989). Why is word recognition impaired by disorientation while the identification of single letters is not? Journal of Experimental Psychology: Human Perception \& Performance, 15, 153-163.

Lachmann, T., \& van Leeuwen, C. (2004). Negative congruence effects in letter and pseudo-letter recognition: The role of similarity and response conflict. Cognitive Processing, 5, 239-248.

Martens, V. E. G., \& DE Jong, P. F. (2006). The effect of visual word features on the acquisition of orthographic knowledge. Journal of Experimental Child Psychology, 93, 337-356.

Marzi, T., \& Viggiano, M. P. (2007). Interplay between familiarity and orientation in face processing: An ERP study. International Journal of Psychophysiology, 65, 182-192.

Maurer, U., Brem, S., Bucher, K., \& Brandeis, D. (2005). Emerging neurophysiological specialization for letter strings. Journal of Cognitive Neuroscience, 17, 1532-1552.

McCandliss, B. D., Cohen, L., \& Dehaene, S. (2003). The visual word form area: Expertise for reading in the fusiform gyrus. Trends in Cognitive Sciences, 7, 293-299.

McClelland, J. L., \& Rumelhart, D. E. (1981). An interactive activation model of context effects in letter perception: Part 1. An account of basic findings. Psychological Review, 88, 375-407.

Mecacci, L., \& Salmaso, D. (1984). Evoked potentials by letters in printed and script forms. Perceptual \& Motor Skills, 59, 227-232.

Milivojevic, B., Clapp, W. C., Johnson, B. W., \& Corballis, M. C. (2003). Turn that frown upside down: ERP effects of thatcherization of misorientated faces. Psychophysiology, 40, 967-978.

Misra, M., \& Holcomb, P. J. (2003). Event-related potential indices of masked repetition priming. Psychophysiology, 40, 115-130.

Nobre, A. C., Allison, T., \& McCarthy, G. (1994). Word recognition in the human inferior temporal lobe. Nature, 372, 260-263.

OldFIELD, R. C. (1971). The assessment and analysis of handedness: The Edinburgh Inventory. Neuropsychologia, 9, 97-113.

Pelli, D. G., Burns, C. W., Farell, B., \& Moore-Page, D. C. (2006). Feature detection and letter identification. Vision Research, 46, 46464674.

Pelli, D. G., Farell, B., \& Moore, D. C. (2003). The remarkable inefficiency of word recognition. Nature, 423, 752-756.

Perea, M., Dunabeitia, J. A., \& Carreiras, M. (2008). R34d1ng W0rd5 w1th numb3r5. Journal of Experimental Psychology: Human Perception \& Performance, 34, 237-241.

Perrin, F., Pernier, J., Bertrand, O., \& Echallier, J. F. (1989). Spherical splines for scalp potential and current density mapping. Electroencephalography \& Clinical Neurophysiology, 72, 184-187.

Petersen, S. E., Fox, P. T., Snyder, A. Z., \& Raichle, M. E. (1990). Activation of extrastriate and frontal cortical areas by visual words and word-like stimuli. Science, 249, 1041-1044.

Petit, J.-P., \& Grainger, J. (2002). Masked partial priming of letter perception. Visual Cognition, 9, 337-353.

Petit, J.-P., Midgley, K. J., Holcomb, P. J., \& Grainger, J. (2006). On the time course of letter perception: A masked priming ERP investigation. Psychonomic Bulletin \& Review, 13, 674-681.

PolK, T. A., \& FARAH, M. J. (1997). A simple common contexts explanation for the development of abstract letter identities. Neural Computation, 9, 1277-1289.

POLK, T. A., \& FARAH, M. J. (1998). The neural development and organization of letter recognition: Evidence from functional neuroimaging, computational modeling, and behavioral studies. Proceedings of the National Academy of Sciences, 95, 847-852.

Polk, T. A., Stallcup, M., Aguirre, G. K., Alsop, D. C., D'Esposito, M., Detre, J. A., \& FARAH, M. J. (2002). Neural specialization for letter recognition. Journal of Cognitive Neuroscience, 14, 145-159.

Posner, M. I., \& BoIEs, S. J. (1969). Retention of visual and name codes of single letters. Journal of Experimental Psychology, 79, 1-16.

Posner, M. I., \& KeELE, S. W. (1967). Decay of visual information from a single letter. Science, 158, 137-139. 
Proverbio, A. M., Vecchi, L., \& Zani, A. (2004). From orthography to phonetics: ERP measures of grapheme-to-phoneme conversion mechanisms in reading. Journal of Cognitive Neuroscience, 16, 301-317.

RugG, M. D., \& Coles, M. G. H. (1995). Electrophysiology of mind: Event-related brain potentials and cognition. New York: Oxford University Press.

Schendan, H. E., Ganis, G., \& Kutas, M. (1998). Neurophysiological evidence for visual perceptual categorization of words and faces within $150 \mathrm{~ms}$. Psychophysiology, 35, 240-251.

Selfridge, O. (1959). Pandemonium: A paradigm for learning. In D. V. Blake \& A. M. Uttley (Eds.), Proceedings of the Symposium of Mechanisation of Thought Processes (pp. 511-529). London: H. M. Stationary Office.

Shin, E., Fabiani, M., \& Gratton, G. (2006). Multiple levels of stimulus representation in visual working memory. Journal of Cognitive Neuroscience, 18, 844-858.

Tarkiainen, A., Helenius, P., Hansen, P. C., Cornelissen, P. L., \& Salmelin, R. (1999). Dynamics of letter string perception in the human occipitotemporal cortex. Brain, 122, 2119-2131.

Townsend, J. T., \& Ashby, F. G. (1982). Experimental test of contemporary mathematical models of visual letter recognition. Journal of Experimental Psychology: Human Perception \& Performance, 8, 834-864.

van Leeuwen, C., \& Lachmann, T. (2004). Negative and positive congruence effects in letters and shapes. Perception \& Psychophysics, 66, 908-925.

Vinckier, F., Dehaene, S., Jobert, A., Dubus, J.-P., Sigman, M., \& CoHen, L. (2007). Hierarchical coding of letter strings in the ventral stream: Dissecting the inner organization of the visual word-form system. Neuron, 55, 143-156.

Wong, A. C.-N., Gauthier, I., Woroch, B., DeBuse, C., \& Curran, T. (2005). An early electrophysiological response associated with expertise in letter perception. Cognitive, Affective, \& Behavioral Neuroscience, 5, 306-318.

Ziegler, J. C., Ferrand, L., Jacobs, A. M., Rey, A., \& Grainger, J.
(2000). Visual and phonological codes in letter and word recognition: Evidence from incremental priming. Quarterly Journal of Experimental Psychology, 53A, 671-692.

\section{NOTES}

1. In some previous studies (e.g., Arguin \& Bub, 1995; Jacobs \& Grainger, 1991; Ziegler et al., 2000), nonletter stimuli have not been well controlled spatially or visually for comparison with letters (consider * in comparison with $m$ ). Here, we used a set of false fonts that were created by rearranging the parts of letters into new letter-like characters and thus controlled for elements of the letters (e.g., curves and straight lines), spatial information, and luminescence (Grossi \& Coch, 2005).

2 . The results of separate analyses of data recorded at midline sites were consistent with the results reported below for lateral and medial sites. Because the midline analyses did not add any information or modify the findings at lateral and medial sites, since the most posterior midline site recorded from was $\mathrm{Pz}$ (and the key effects were posterior to $\mathrm{Pz}$ ), and for efficiency and readability, we have not included the midline analysis results below. As in the lateral and medial site mean amplitude analyses, the critical match $\times$ condition interaction was not significant at the three midline sites in either the P150 (120-180 msec, $p=.57)$ or the P260 (220-300 msec, $p=.52)$ window.

3. Note that incorrect trials were not included in ERP averages, so that the ERP results likely were unaffected by this behavioral finding.

4. Because we did not use combined letter-false-font prime-target pairs, we were not able to evaluate whether our false fonts could prime our letters or vice versa. Although not the focus of the present investigation, such a cross-font comparison could address the specificity of the P260 priming effect and the nature of our false fonts from another perspective in future research.

5 . We thank a reviewer for this suggestion.

(Manuscript received July 30, 2008;

revision accepted for publication February 13, 2009.) 